\title{
Thermodynamic properties of harmonically trapped $D$-dimensional ideal gases within generalized exclusion statistics
}

\author{
S. Sevinçli, B. Tanatar* \\ Department of Physics, Bilkent University, Bilkent, 06800 Ankara, Turkey \\ Received 11 June 2007; accepted 19 June 2007 \\ Available online 27 June 2007 \\ Communicated by V.M. Agranovich
}

\begin{abstract}
We consider an ideal gas trapped with harmonic potential and obeying the generalized exclusion statistics. We analytically calculate the density of states from which the thermodynamic properties of the gas are calculated for a general energy spectrum $\varepsilon(p)=a p^{s}$ in $D$-dimensional space. Internal energy and the specific heat as a function of temperature are evaluated numerically for different dimensions and energy-momentum dispersion relations. In particular, we show that the specific heat is independent of the statistical parameter $g$ for the constant density of states regime which corresponds $D=1$ and $s=2$ for this system.
\end{abstract}

(C) 2007 Elsevier B.V. All rights reserved.

PACS: 05.30.-d; 05.30.Pr; 05.70.-a; 05.70.Ce

Keywords: Bose gases; Low dimensional; Density profiles

\section{Introduction}

Particle statistics drastically affect thermodynamic properties of quantum many-body systems. Fundamental particles obey either Bose-Einstein or Fermi-Dirac statistics and these bosonic and fermionic nature successfully describe many physical phenomena.

In 1991 Haldane introduced the so-called fractional statistics or generalized exclusion statistics (GES) after observation of particles in one- and two-dimensional systems [1]. These particles have intermediate statistics between bosons and fermions. Haldane formulated the generalized Pauli exclusion principle. He defined the GES through the linear relation

$$
\Delta d_{i}=-\sum_{j} g_{i j} \Delta N_{j}
$$

where $\Delta d_{i}$ is the change of the available single-particle states number, $\Delta N_{j}$ is the change of the particle numbers at fixed size and boundary conditions and $i, j$ indicate different particle species.

By adopting a state-counting definition, Wu proposed the occupation number distribution of particles which obey fractional statistics [2]. He interpolated the number of quantum states for bosons and fermions and defined the statistical weight of $N$ identical particles occupying a group of $G$ states as

$$
W=\prod_{i} \frac{\left[G_{i}+\left(N_{i}-1\right)(1-g)\right] !}{N_{i} !\left[G_{i}-g N_{i}-(1-g)\right] !} .
$$

\footnotetext{
* Corresponding author.

E-mail address: tanatar@fen.bilkent.edu.tr (B. Tanatar).
} 
This weight corresponds to bosons when $g=0$ and to fermions when $g=1$. The parameter $g$ characterizes the generalized Pauli principle since the occupation number for single-particle state has a maximum value of $1 / \mathrm{g}$.

There has been a growing interest in GES since Haldane's formulation. It has been shown that the anyon gas in the lowest Landau level satisfies the GES [1-4]. Several researchers have studied the thermodynamic properties of the ideal $g$-on gas using different methods [5-11]. Very different systems have been also studied such as 1D and 2D Bose gas [12,13], ideal and interacting quantum gases [14,15]. Hansson et al. [13] established that a 2D Bose gas with a repulsive delta-function interaction obeys exclusion statistics and is equivalent to an ideal exclusion statistics gas by a direct counting of states. Different descriptions for the occupation number were proposed [16,17]. Recently, Bhaduri et al. [18] calculated the chemical potential and the energy of the gas of neutral fermionic atoms by assuming the system to be an ideal gas which obeys Haldane-Wu statistics. They also presented the results for harmonically trapped gas. In a very recent paper, Potter et al. [19] explored the thermodynamics of the generalized CalogeroSutherland model in $D \geqslant 1$ dimensions and also studied the effects of soft container walls. In two recent papers [20,21], it is shown that $1 \mathrm{D}$ interacting anyons and bosons in the strong coupling regime are properly described by GES and the quasiparticle excitations of these two systems also obey GES. Batchelor et al. showed that the distribution profiles of strongly interacting 1D anyon gas and Bose gas are equivalent to the most probable distribution profiles of ideal particles obeying fractional statistics.

In this Letter, we calculate the thermodynamic properties of the ideal gas within the generalized exclusion statistics ( $g$-on gas) trapped with a harmonic potential in $D$ dimension. Other than the above related works we are also motivated by a recent proposal by Paredes et al. [22] to observe fractional statistics of anyons in a system of ultracold bosonic atoms in a rapidly rotating trap. Generally, harmonic potentials created by magneto-optical techniques, are used for trapping the ultracold Bose gases. We assume that the particles have the energy spectrum of the form $\varepsilon(p)=a p^{s}$ and neglect the spin degree of freedom. We calculated the specific heat of the ideal trapped $g$-on gas in several spatial dimensions and different dispersion relations. It is important to establish the thermodynamic properties of ideal gas systems before tackling the interaction effects.

\section{Density of states and thermodynamic quantities}

The density of states (DOS) for the particles in a harmonic potential in the $D$-dimensional space is calculated as

$$
D(\varepsilon)=\int \frac{d^{D} r d^{D} p}{(2 \pi \hbar)^{D}} \delta\left[\varepsilon-\left(\varepsilon(p)+\frac{1}{2} m \omega^{2} r^{2}\right)\right] .
$$

Here it is assumed that the system is in the thermodynamic limit, i.e. the number if particles $N \rightarrow \infty$, the volume $V \rightarrow \infty$, such that the number density $N / V$ is constant. Thus, possible corrections to the DOS when the number of particles are finite are not included. Furthermore, because we have an inhomogeneous system due to the harmonic trap, the volume has to be understood as $V=\omega^{-D}$ in a $D$-dimensional space [23].

Using the properties of the delta-function one can perform the $r$-integral,

$$
D(\varepsilon)=\frac{S_{D}^{2} 2^{\frac{D-2}{2}} \varepsilon^{\frac{D-2}{2}}}{\left(2 \pi m^{1 / 2}\right)^{D}(\hbar \omega)^{D}} \int_{0}^{(\varepsilon / a)^{1 / s}} d p p^{D-1}\left(1-\frac{a p^{s}}{\varepsilon}\right),
$$

where $S_{D}=\frac{2 \pi^{D / 2}}{\Gamma(D / 2)}$ is the $D$-dimensional surface area. Finally, the DOS is

$$
D(\varepsilon)=\frac{S_{D}^{2} 2^{\frac{D-2}{2}}}{\left(2 \pi m^{1 / 2}\right)^{D} s a^{D / s}} \frac{\Gamma(D / s) \Gamma(D / 2)}{\Gamma\left(\frac{D}{s}+\frac{D}{2}\right)} \frac{\varepsilon^{\frac{D}{s}+\frac{D-2}{2}}}{(\hbar \omega)^{D}} i .
$$

The distribution function in generalized exclusion statistics is given as [2]

$$
f(\varepsilon)=\frac{1}{w+g}
$$

where $w$ obeys the relation

$$
w^{g}(1+w)^{1-g}=\exp [\beta(\varepsilon-\mu)]
$$

in which $\beta=1 / T$ (temperature is in units of Boltzmann constant) and $\mu$ is the chemical potential. One can see that from the above equation for $g=0$ (1), the distribution function reduces to the bosonic (fermionic) form. The average occupation number at the zero temperature is

$$
f(\varepsilon)= \begin{cases}1 / g & (\varepsilon-\mu) \leqslant 0 \\ 0 & (\varepsilon-\mu) \geqslant 0\end{cases}
$$


For a given density of states $D(\varepsilon)$ we have

$$
N=\int_{0}^{\infty} f(\varepsilon) D(\varepsilon) d \varepsilon, \quad E=\int_{0}^{\infty} f(\varepsilon) \varepsilon D(\varepsilon) d \varepsilon
$$

for the number of particles $N$ in the system and the total energy $E$, respectively.

At $T=0$, the Fermi energy is found as

$$
\varepsilon_{F}=\left[\frac{N g}{\gamma}\left(\frac{D}{s}+\frac{D}{2}\right)\right]^{1 /\left(\frac{D}{s}+\frac{D}{2}\right)}
$$

where $\gamma=\frac{S_{D}^{2} 2^{\frac{D-2}{2}}}{\left(2 \pi m^{1 / 2}\right)^{D} s a^{D / s}} \frac{\Gamma(D / s) \Gamma(D / 2)}{\Gamma\left(\frac{D}{s}+\frac{D}{2}\right)} \frac{1}{(\hbar \omega)^{D}}$. The energy per particle is

$$
\frac{E}{N}=\frac{\left(\frac{D}{s}+\frac{D}{2}\right)}{\left(\frac{D}{s}+\frac{D+2}{2}\right)} \varepsilon_{F} .
$$

At finite temperature energy per particle can be calculated similarly. Converting the integration variable $\varepsilon$ to $w$, for $N$ and $E$ we get

$$
1=\left(\frac{D}{s}+\frac{D}{2}\right)\left(\frac{T}{\epsilon_{F}}\right)^{\left(\frac{D}{s}+\frac{D}{2}\right)} \int_{w_{0}}^{\infty} \frac{d w}{w(1+w)}\left\{\ln \left[\left(\frac{w}{w_{0}}\right)^{g}\left(\frac{1+w}{1+w_{0}}\right)^{1-g}\right]\right\}^{\left(\frac{D}{s}+\frac{D-2}{2}\right)}
$$

and

$$
\frac{E}{N \epsilon_{F}}=\left(\frac{D}{s}+\frac{D}{2}\right)\left(\frac{T}{\epsilon_{F}}\right)^{\left(\frac{D}{s}+\frac{D+2}{2}\right)} \int_{w_{0}}^{\infty} \frac{d w}{w(1+w)}\left\{\ln \left[\left(\frac{w}{w_{0}}\right)^{g}\left(\frac{1+w}{1+w_{0}}\right)^{1-g}\right]\right\}^{\left(\frac{D}{s}+\frac{D}{2}\right)}
$$

where $w_{0}$ is the value of $w$ at $\varepsilon=0, w_{0}^{g}\left(1+w_{0}\right)^{1-g}=\exp (-\beta \mu)$ and $\epsilon_{F}$ is the Fermi energy at $g=1$. One can calculate the specific heat readily using $C_{v}=\frac{\partial E}{\partial T}$.

$$
C_{v}=\int d \varepsilon D(\varepsilon) \varepsilon \frac{\partial}{\partial T} \frac{1}{w+g}
$$

and after some algebra specific heat per particle is given as

$$
C_{v}=\int d \varepsilon D(\varepsilon) \varepsilon\left[\frac{\varepsilon-\mu}{T^{2}}+\frac{1}{T} \frac{\partial \mu}{\partial T}\right] \frac{w(1+w)}{(w+g)^{3}} .
$$

We can find the $\frac{\partial \mu}{\partial T}$ using Eq. (12) and the Leibniz integral rule as

$$
\frac{\partial \mu}{\partial T}=-\ln \left[w_{0}^{g}\left(1+w_{0}\right)^{1-g}\right]-\frac{\left(\frac{D}{s}+\frac{D}{2}\right)}{\left(\frac{D}{s}+\frac{D-2}{2}\right)} \frac{\int_{w_{0}}^{\infty} \frac{d w}{w(1+w)}\left\{\ln \left[\left(\frac{w}{w_{0}}\right)^{g}\left(\frac{1+w}{1+w_{0}}\right)^{1-g}\right]\right\}^{\left(\frac{D}{s}+\frac{D-2}{2}\right)} \frac{d w}{w(1+w)}\left\{\ln \left[\left(\frac{w}{w_{0}}\right)^{g}\left(\frac{1+w}{1+w_{0}}\right)^{1-g}\right]\right\}^{\left(\frac{D}{s}+\frac{D-4}{2}\right)}}{\int_{0}} .
$$

Then the specific heat can be written as

$$
\begin{aligned}
& \frac{C_{v}}{N}=\left(\frac{D}{s}+\frac{D}{2}\right)\left(\frac{T}{\epsilon_{F}}\right)^{\left(\frac{D}{s}+\frac{D}{2}\right)} \int_{w_{0}}^{\infty} \frac{d w}{(w+g)^{2}}\left\{\ln \left[\left(\frac{w}{w_{0}}\right)^{g}\left(\frac{1+w}{1+w_{0}}\right)^{1-g}\right]\right\}^{\left(\frac{D}{s}+\frac{D}{2}\right)} \\
& \times\left[\ln \left[\left(\frac{w}{w_{0}}\right)^{g}\left(\frac{1+w}{1+w_{0}}\right)^{1-g}\right]-\frac{\left(\frac{D}{s}+\frac{D}{2}\right) \int_{w_{0}}^{\infty} \frac{d w}{w(1+w)}\left\{\ln \left[\left(\frac{w}{w_{0}}\right)^{g}\left(\frac{1+w}{1+w_{0}}\right)^{1-g}\right]\right\}^{\left(\frac{D}{s}+\frac{D-2}{2}\right)}}{\left(\frac{D}{s}+\frac{D-2}{2}\right) \int_{w_{0}}^{\infty} \frac{d w}{w(1+w)}\left\{\ln \left[\left(\frac{w}{w_{0}}\right)^{g}\left(\frac{1+w}{1+w_{0}}\right)^{1-g}\right]\right\}^{\left(\frac{D}{s}+\frac{D-4}{2}\right)}}\right] .
\end{aligned}
$$

To find the general relation between the pressure and the total energy we consider the thermodynamic potential $\Omega$,

$$
\Omega=-P V=-T \ln Z
$$

where the grand canonical partition function is given as

$$
Z=\sum_{\left\{N_{i}\right\}} W\left(\left\{N_{i}\right\}\right) \exp \left[-\beta \sum_{i} N_{i}\left(\varepsilon_{i}-\mu\right)\right]
$$


In terms of the distribution in Eq. (6) the thermodynamic potential is given by

$$
\Omega=-P V=-T \sum_{i} G_{i} \ln \frac{1+(1-g) f_{i}}{1-g f_{i}}=-T \sum_{i} G_{i} \ln \left[1+\frac{1}{w}\right] .
$$

In the thermodynamic limit a sum can be replaced by an integration, then the pressure is

$$
\frac{P}{T}=\gamma \int_{0}^{\infty} d \varepsilon \varepsilon^{\left(\frac{D}{s}+\frac{D}{2}\right)} \ln \left[1+\frac{1}{w}\right]
$$

Integrating by parts we obtain

$$
\frac{P}{T}=\frac{\beta}{\left(\frac{D}{s}+\frac{D}{2}\right)} \int_{0}^{\infty} f(\varepsilon) \varepsilon D(\varepsilon) d \varepsilon, \quad P V=\frac{E}{\left(\frac{D}{s}+\frac{D}{2}\right)} .
$$

This is the so-called Bernoulli equation for a harmonically trapped ideal gas obeying generalized exclusion statistics. In the above derivation, although we have used the thermodynamic variables pressure $P$ and volume $V$ in the definition of the grand potential, their meaning for trapped systems deserves special attention as we have already remarked. As discussed by RomeroRochin [23] recently, the volume can be identified as $V=\omega^{-D}$ in $D$-dimensional space and its conjugate variable harmonic pressure is $P=-(\partial \Omega / \partial V)_{T, \mu}$.

Finally, the entropy of the system can be found using the relation $S=\frac{E}{T}+\ln Z-\frac{N \mu}{T}$. Then the entropy is obtained as

$$
S=\frac{E}{T}\left[1+\frac{1}{\left(\frac{D}{S}+\frac{D}{2}\right)}\right]-\frac{N \mu}{T} .
$$

The above result is slightly different from the entropy expression found by Yang [11] since they performed calculations for the uniform gas.

\section{Results and discussion}

Based on the theoretical formulation of the thermodynamic quantities of an ideal $g$-on gas in $D$-dimensions, we now present some illustrative examples.

For a 3D space and when $s=2$, the total energy per particle as a function of temperature is shown in Fig. 1. At low temperatures, the energy increases with $g$ and at high temperature all curves have the same slope. The specific heat as a function of temperature is shown in Fig. 2. The specific heat increases with statistical parameter $g$ at low temperatures and decreases at high temperatures

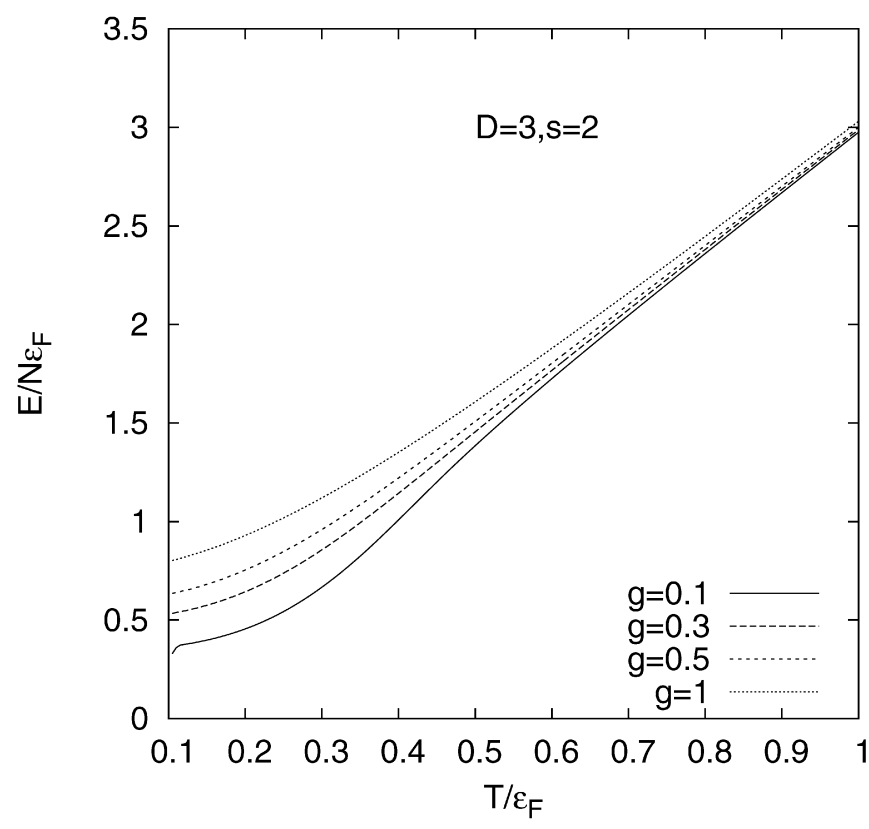

Fig. 1. Energy per particle as a function of temperature in $3 \mathrm{D}$ for various values of the statistical parameter $g$.

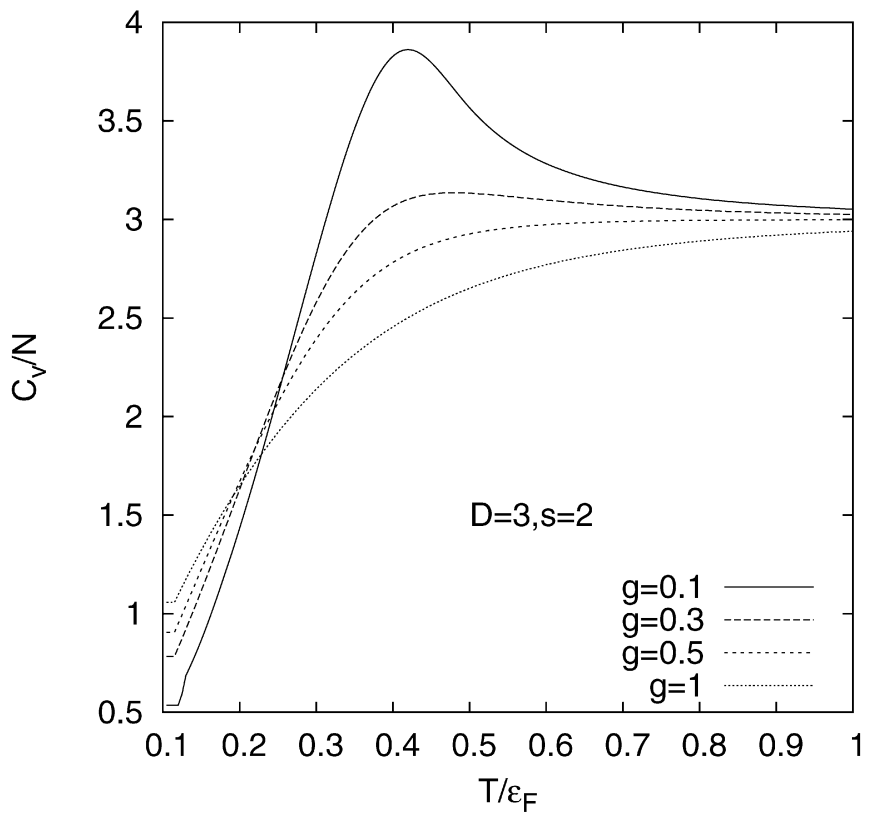

Fig. 2. Specific heat per particle as a function of temperature in $3 \mathrm{D}$ for various values of the statistical parameter $g$. 


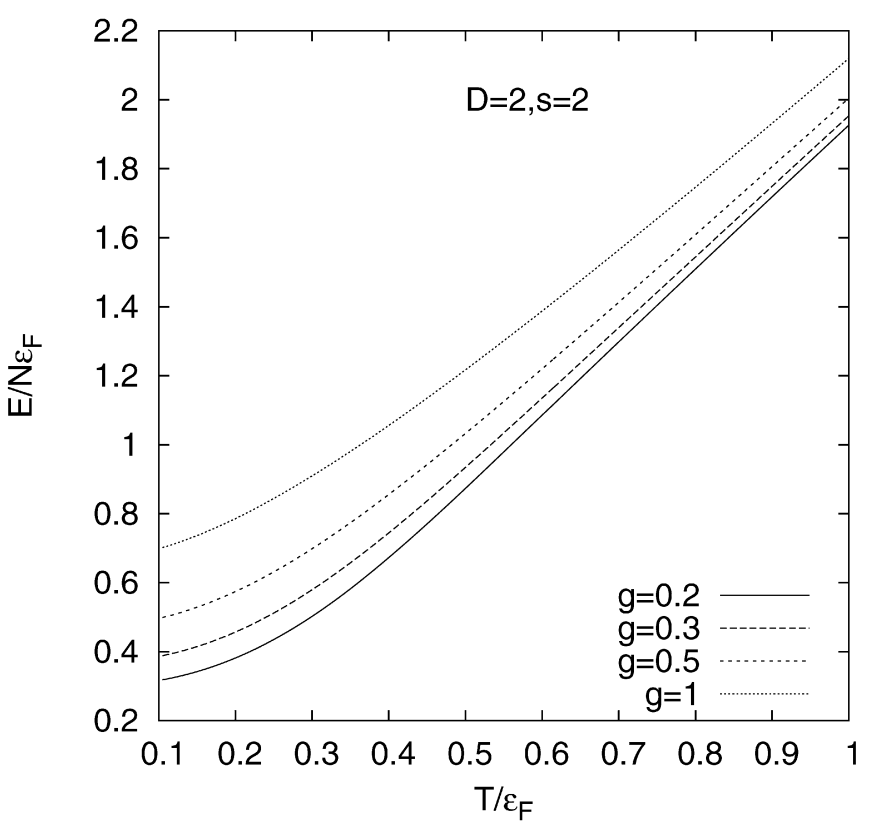

Fig. 3. Energy per particle as a function of temperature in $2 \mathrm{D}$ for various values of the statistical parameter $g$.

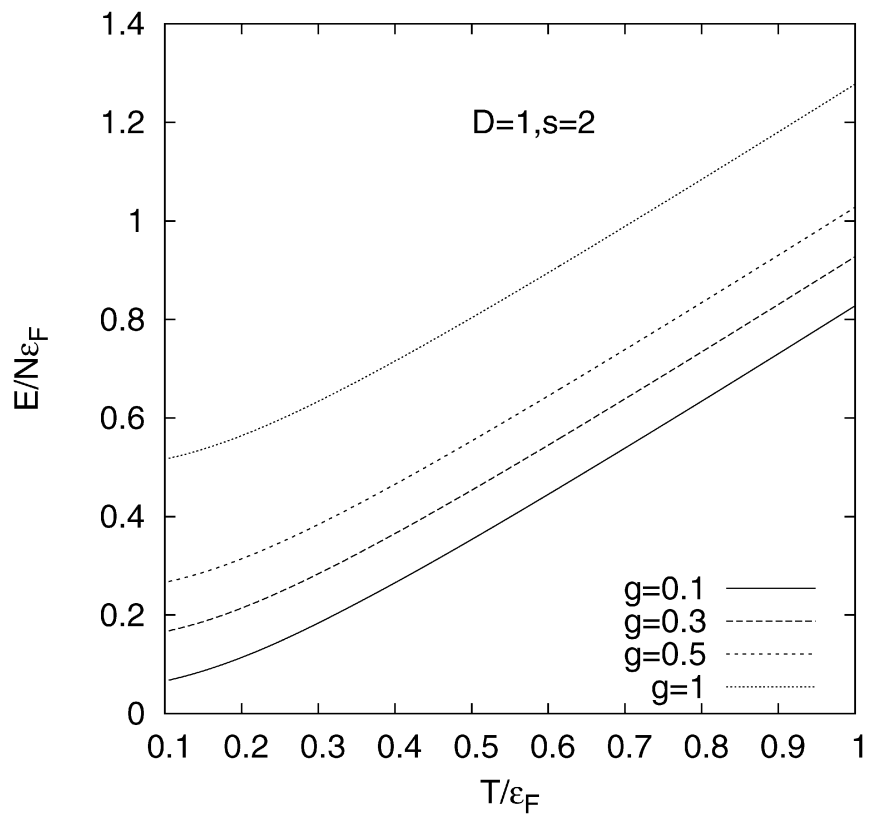

Fig. 5. Energy per particle as a function of temperature in $1 \mathrm{D}$ for various values of the statistical parameter $g$.

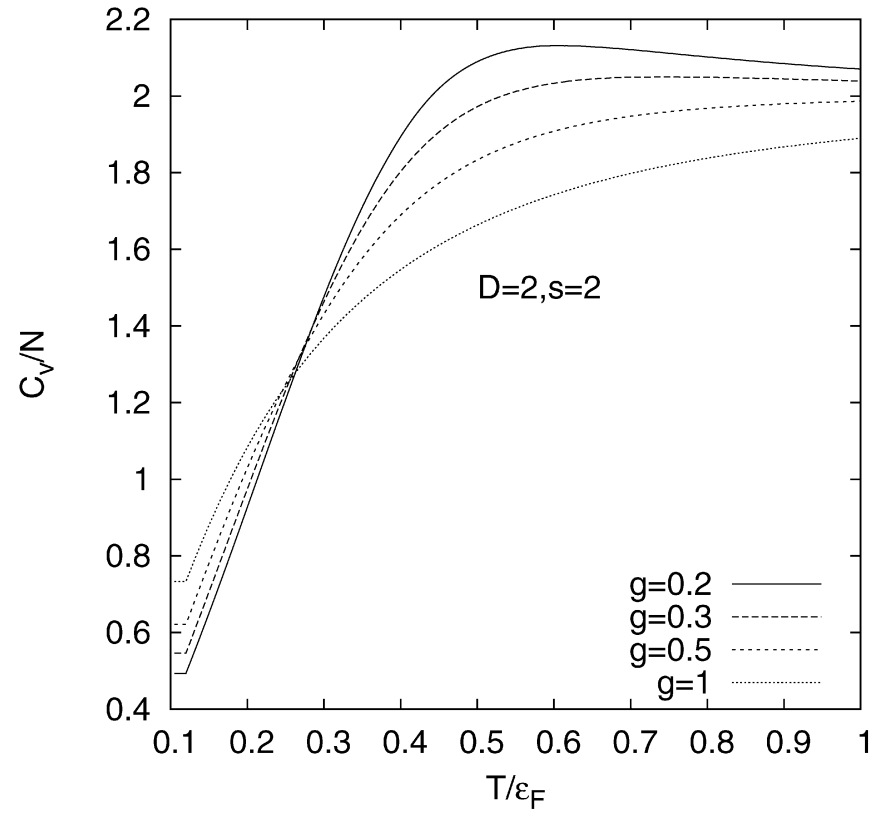

Fig. 4. Specific heat per particle as a function of temperature in $2 \mathrm{D}$ for various values of the statistical parameter $g$.

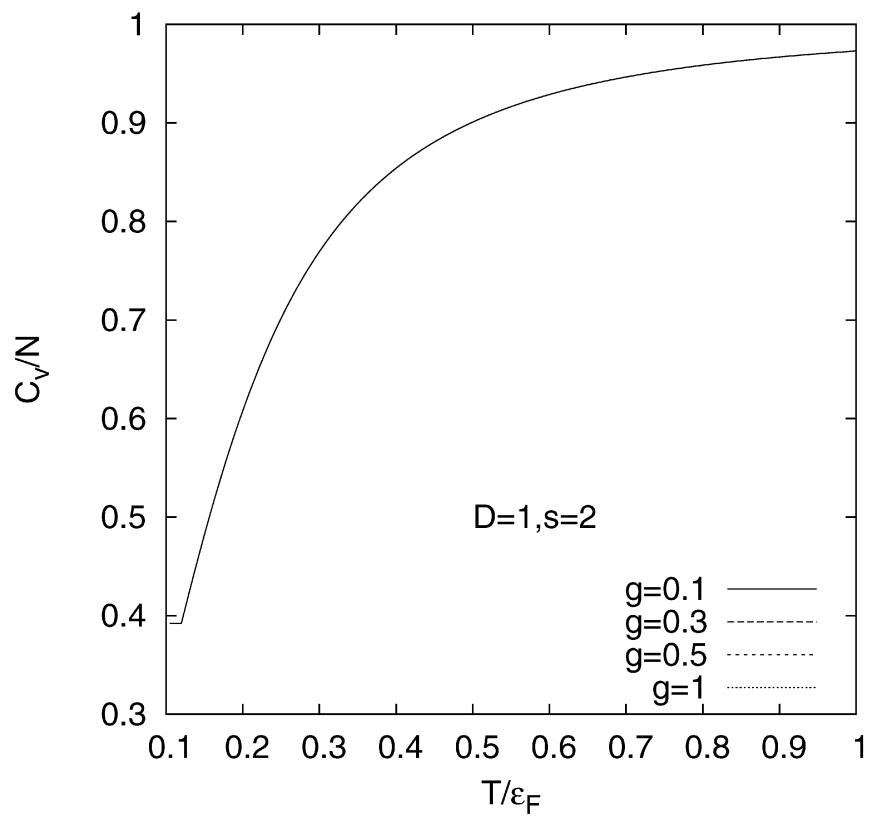

Fig. 6. Specific heat per particle as a function of temperature in 1D. In this case specific heat does not depend on the statistical parameter $g$.

reaching eventually the classical value in the high temperature regime. One can also see that it starts to exhibit bosonic behavior as $g$ approaches 0 . The energy and the specific heat for a 2D system are similar to 3D ones as shown in Figs. 3 and 4, respectively.

Figs. 5 and 6 show the interesting situation where the density of states is constant $(D=1, s=2)$ of the energy and specific heat, respectively. We observe in Fig. 5 that the energy curves for various $g$ values differ by a temperature independent constant and in Fig. 6 that the specific heat does not depend on the statistical parameter $g$. Similarly, the entropy $S$ also becomes independent of $g$. It is known that for a constant density of states differences between bosons and fermions disappear, and as far as the thermodynamics is concerned the systems are said to be equivalent. For an ideal uniform system the constant density of states and consequently the same specific heat for bosons and fermions takes place in 2D for quadratic dispersion [9,24].

Finally, for a system which has energy dispersion $\varepsilon \sim p$ in 1D the energy and specific heat are shown in Figs. 7 and 8, respectively. Interestingly, we have the same total energy relation with the uniform system that has energy dispersion $\varepsilon \sim p^{2}$ in 3D [9]. 


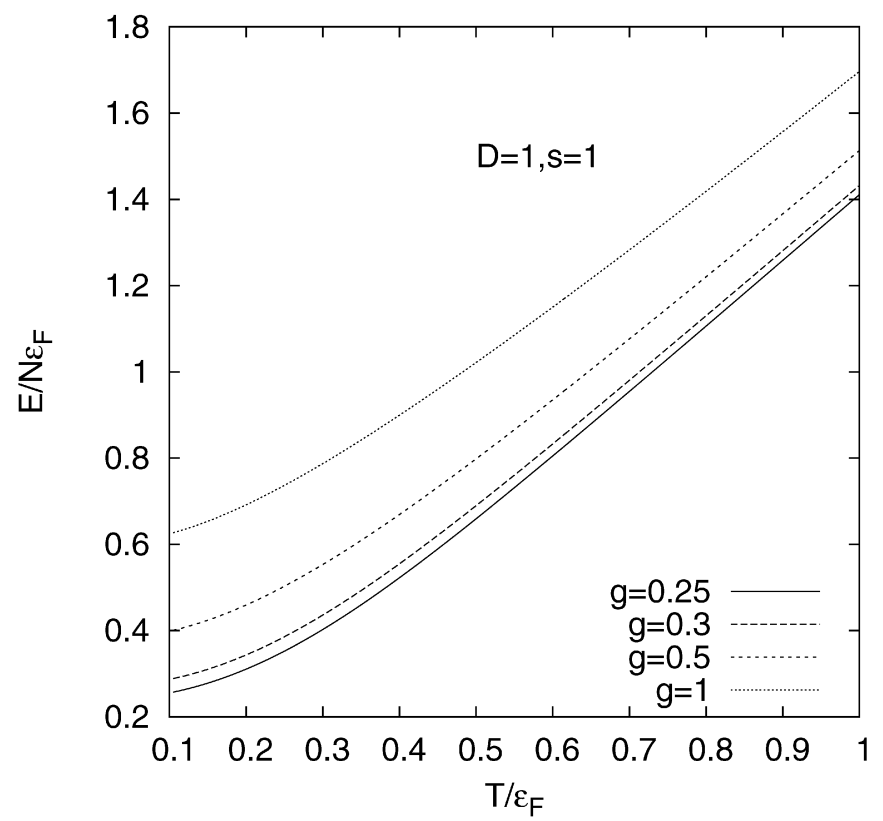

Fig. 7. Energy per particle as a function of temperature in 1D and dispersion $\sim p$.

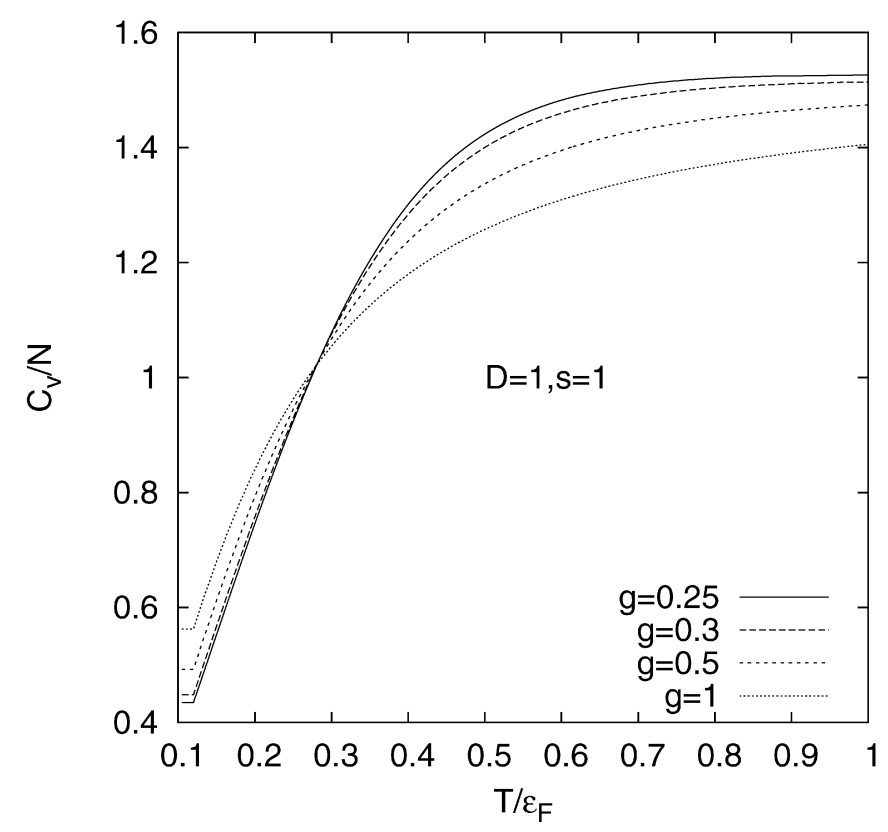

Fig. 8. Specific heat per particle as a function of temperature in 1D and dispersion $\sim p$.

There is a critical temperature at which the specific heat does not depend on $g$. It means that there is no difference between bosons and fermions at this specific temperature. Below this temperature the entropy increases by $g$, but it decreases above this critical temperature. Similar behavior can be observed for other spatial dimensions and dispersion relations.

To summarize, we have considered ideal gases trapped by a harmonic oscillator potential in $D$-dimensions and obeying the generalized exclusion statistics. Thermodynamic properties such as energy and specific heat as functions of temperature are calculated numerically for various spatial dimensions and single-particle energies. Illustrative examples are presented for various values of the statistical parameter $g$. As trapped systems of atomic gases are of growing interest in recent years, we expect our results will be useful for further studies.

\section{Acknowledgements}

We acknowledge support for this work by TUBITAK (106T052) and TUBA, and thank Dr. M.Ö. Oktel for fruitful discussions.

\section{References}

[1] F.D.M. Haldane, Phys. Rev. Lett. 67 (1991) 937.

[2] Y.-S. Wu, Phys. Rev. Lett. 73 (1994) 922.

[3] A.K. Rajagopal, Phys. Rev. Lett. 74 (1995) 1048.

[4] M.T. Batchelor, X.-W. Guan, N. Oelkers, Phys. Rev. Lett. 96 (2006) 210402.

[5] K. Iguchi, Phys. Rev. Lett. 78 (1997) 3233.

[6] G.S. Joyce, S. Sarkar, J. Spalek, K. Byczuk, Phys. Rev. B 53 (1996) 990.

[7] S.B. Isakov, S. Ouvry, J. Phys. A: Math. Gen. 29 (1996) 7401.

[8] S.B. Isakov, D.P. Arovas, J. Myrheim, A.P. Polychronakos, Phys. Lett. A 212 (1996) 299.

[9] T. Aoyama, Eur. Phys. J. B 20 (2001) 123.

[10] G. Su, M. Suzuki, Eur. Phys. J. B 5 (1998) 577.

[11] H.S. Yang, B. Lee, C. Park, J. Kor. Phys. Soc. 30 (1996) 14.

[12] R.K. Bhaduri, M.V.N. Murthy, M.N. Tran, J. Phys. B: At. Mol. Opt. Phys. 35 (2002) 2817.

[13] T.H. Hansson, J.M. Leinaas, S. Viefers, Phys. Rev. Lett. 86 (2001) 2930.

[14] D.V. Anghel, J. Phys. A: Math. Gen. 35 (2002) 7255.

[15] D.-V. Anghel, Phys. Rev. E 62 (2000) 7658.

[16] W. Bao-Quan, W. Yu-Peng, Chin. Phys. Lett. 22 (2005) 824.

[17] F. Büyükkılıç, H. Uncu, D. Demirhan, Eur. Phys. J. B 35 (2003) 111.

[18] R.K. Bhaduri, M.V.N. Murthy, M.K. Srivastava, J. Phys. B: At. Mol. Opt. Phys. 40 (2007) 1775.

[19] G.G. Potter, G. Muller, M. Karbach, cond-mat/0610400.

[20] M.T. Batchelor, X.-W. Guan, N. Oelkers, Phys. Rev. Lett. 96 (2006) 210402.

[21] M.T. Batchelor, X.-W. Guan, Phys. Rev. B 74 (2006) 195121.

[22] B. Paredes, P. Fedichev, J.I. Cirac, P. Zoller, Phys. Rev. Lett. 87 (2001) 010402.

[23] V. Romero-Rochin, Phys. Rev. Lett. 94 (2005) 130601.

[24] M.H. Lee, Phys. Rev. E 55 (1997) 1518. 